\title{
Análisis de supervivencia de cáncer de recto no metastásico en Bogotá (Colombia)
}

\author{
Pedro Luis Ramos Guette
}

Correos electrónicos: pramos@oncocareltda,pramos152@yahoo.com

\section{Resumen}

Objetivos: evaluar la supervivencia global y libre de enfermedad en cáncer de recto no metastásico y la asociación con factores pronósticos.

Materiales y métodos: se trata de un estudio de cohorte bidireccional; se registró la información de las características clínicas, demográficas y los tiempos de recaída y muerte por cáncer u otras causas de una cohorte de 127 pacientes con cáncer de recto durante el período 2003-2012. Los pacientes fueron seguidos en intervalos cada 4 a 6 meses, en los cuales se documentaba el estado vital y la presencia de recaídas locales o sistémicas de la enfermedad. Se realizaron análisis descriptivos y se estimaron funciones de supervivencia usando el método de Kaplan-Meier. Se llevó a cabo análisis de regresión de Cox para identificar factores asociados a la supervivencia.

Resultados: se identificaron 127 pacientes con cáncer de recto. La mediana de seguimiento fue 74 meses, la edad promedio fue 64 años, del sexo femenino fue el $51,18 \%$, se presentó en estadio avanzado III el $66,29 \%$. Se dio un $23,62 \%$ de recaídas y se observó un $18,11 \%$ de muertes. En análisis multivariado, se evidenció asociación del estadio clínico avanzado III con menor sobrevida libre de enfermedad y global, HR 3,40 (IC95\% 1,36-8,47) y 2,51 (IC95\% 1,09-5,79), respectivamente. Los otros factores: grado histológico, tipo histológico, estado funcional, tipo de quimioterapia y respuesta a la quimiorradioterapia neoadyuvante, no se relacionaron con la supervivencia libre de enfermedad y global.

Conclusión: los tiempos de supervivencia libre de enfermedad y global observados en la cohorte se asocian con el estadio clínico avanzado, pero no hay relación con el grado histológico, el tipo histológico, el estado funcional, el tipo de quimioterapia y las respuestas a quimiorradioterapia neoadyuvante.

\section{Análisis de supervivencia de cáncer de cérvix no metastásico en Bogotá (Colombia)}

\author{
Pedro Luis Ramos Guette
}

Correos electrónicos: pramos@oncocareltda, pramos152@yahoo.com

\section{Resumen}

\begin{abstract}
Objetivo: conocer la supervivencia global y libre de enfermedad de mujeres con cáncer de cérvix no metastásico.

Materiales y métodos: se trata de un estudio de cohorte bidireccional; en la clínica Oncocare se registró la información de las características clínicas, demográficas y los tiempos de recaída y muerte por cáncer u otras causas de una cohorte de 143 mujeres con cáncer de cérvix durante el período 2003-2012. Las pacientes fueron seguidas en intervalos cada 4 a 6 meses, en los cuales se documentaba el estado vital y la presencia de recaídas locales o sistémicas de la enfermedad. Se realizaron análisis descriptivos y se estimaron funciones de supervivencia usando el método de Kaplan-Meier. Se efectuó análisis de regresión de Cox para identificar factores asociados a la supervivencia.
\end{abstract}

Palabras clave: cáncer de cérvix, pronóstico, análisis de supervivencia.
Resultados: se identificaron 143 pacientes para el análisis descriptivo y analítico. La mediana de seguimiento fue 18,16 meses, la edad promedio de las mujeres fue 54 años, el 95,1\% fue diagnosticado en estadios avanzados IIA a IIIB, el tipo histológico más frecuente fue escamocelular. Se presentó el 13,9\% de recaídas y se reportó un 13,2\% de muertes. Los análisis univariado y multivariado no evidenciaron asociación de ninguno de los factores, como estadio clínico, grado histológico, tipo histológico, estado funcional, respuesta a quimiorradioterapia, con la supervivencia libre de enfermedad y global.

Conclusiones: los tiempos de supervivencia libre de enfermedad y global observados en la cohorte no se relacionan con ningún factor pronóstico analizado y se requiere un seguimiento mayor para tener suficientes eventos que permitan conclusiones definitivas en el análisis. 